\section{atualização}

\author{
Isio Schulz
}

Disciplina de Endocrinologia do Hospital das Clínicas, Faculdade de Medicina da Universidade de São Paulo (HC-FMUSP), São Paulo, SP.

Recebido em 22/12/05

Aceito em 17/01/06

\title{
Tratamento das Dislipidemias - Como e Quando Indicar a Combinação de Medicamentos Hipolipemiantes
}

\section{RESUMO}

Hiperlipidemia combinada familiar (HCF) é a forma mais comum de hiperlipidemia familial e se caracteriza por resistência à insulina, niveis baixos de HDL-C, níveis altos de triglicérides (TGC) e colesterol total associados a vários fenótipos dentro da mesma família. HCF associa-se, também, a um alto risco cardiovascular (RCV), e os níveis-alvo de tratamento das anormalidades lipídicas têm se modificado recentemente. Reduzir os níveis de LDL-C e não HDL-C devem ser os alvos da terapia. Niveis de LDL-C abaixo de $70 \mathrm{mg} / \mathrm{dl}$ têm se mostrado benéficos na RCV em pacientes de alto risco. Várias estatinas com diferentes potências e interações medicamentosas estão disponíveis no mercado. A terapia combinada de estatinas com seqüestradores de ácidos biliares ou ezetimiba pode ser necessária para se alcançar os valores-alvo de LDLC estabelecidos pelas diretrizes. Níveis altos de TGC e baixos de HDL-C devem ser também considerados no tratamento, e freqüentemente somente o uso das estatinas se mostra insuficiente para normalizá-los. A combinação de estatinas com fibratos pode auxiliar para reduzir os niveis de colesterol e aumentar os de HDL-C, mas está associada à maior freqüência de miopatia e toxicidade hepática. Assim, a avaliação cuidadosa dos riscos e benefícios da terapia é recomendável. A associação de estatina e niacina parece ser útil para pacientes com HCF, particularmente por aumentar os níveis de HDL-C, uma vez que tem sido menos relacionada à alta freqüência de miopatia. A niacina pode ser causa de flushings que podem ser reduzidos com o uso de aspirina. $O$ efeito pode também ser minimizado com o uso de formas de liberação lenta (Niaspan). A niacina pode também elevar os níveis de glicemia e ácido úrico. Assim, os riscos e benefícios da associação devem ser avaliados. (Arq Bras Endocrinol Metab 2006;50/2:344-359)

Descritores: Estatina; Fibrato; Niacina; Ezetimiba; Hiperlipidemia combinada; Tratamento

\section{ABSTRACT}

Treatment of Dyslipidemia: How and When to Combine Lipid Lowering Drugs.

Familial combined hyperlipidemia $(\mathrm{FCH})$ is a frequent familial lipid disorder associated with insulin resistance, low HDL cholesterol, high triglycerides and cholesterol levels with variable phenotypes within the same family. FCH is linked to a high risk for cardiovascular diseases. Treatment goals for lipid abnormalities are changing in recent years. Lowering elevated levels of LDL e Non HDL-cholesterol levels are primary targets of therapy. Lower LDL-C than $70 \mathrm{mg} / \mathrm{dL}$ seems to be useful to lower cardiovascular risk in patients with very high risk. Many statins are available, with different potencies and drug interactions. Combination therapy of statins and bile acid sequestrants or ezitimibe may be necessary to further decrease LDL cholesterol levels in order to meet guideline goals. High triglycerides and low HDL cholesterol are also 
important goals in the treatment of these patients, and frequently statins alone are insufficient to normalize the lipid profile. Combination therapy with fibrates will further lower triglycerides and increase HDL cholesterol levels; this combination is also associated with higher incidence of myopathy and liver toxicity; appropriate evaluation of patients' risk and benefits is necessary. Association of statin/niacin seems be very useful in patients with $\mathrm{FCH}$, especially as niacin is the best drug to increase HDL cholesterol; this association is not linked to a higher frequency of myopathy. Niacin causes flushing, that can in part be managed with use of aspirin and extended release forms (Niaspan); niacin also may increase plasma glucose and uric acid levels. Evaluation of risks and benefits for each patient is needed. (Arq Bras Endocrinol Metab 2006;50/2:344-359)

Keywords: Statin; Fibrate; Niacin; Ezetimibe; Combined hiperlipidemia; Treatment

$\mathbf{H}$ PERLIPIDEMIA COMBINADA FAMILIAR (FCH familial combined bypelipidemia) é a forma mais comum de hiperlipidemia familial, sendo a prevalência estimada de $1 \%$ a 3\% na população geral, chegando a até $20 \%$ nos pacientes que apresentaram infarto do miocárdio prematuro (1).

FCH pode apresentar-se com VLDL colesterol (VLDL-C) elevado (tipo IV), LDL colesterol (LDLC) elevado (tipo II a) ou ambos (tipo II b). Pacientes com hiperlipidemia combinada apresentam níveis diminuídos de HDL-colesterol (HDL-C).

Embora as bases metabólicas e genéticas da FCH não tenham sido completamente identificadas, Pajukanta e cols. (2) verificaram que, em 60 famílias, a FCH estava associada a diferentes haplotipos do gene USFl (upstream transcription factor 1). Este gene codifica o fator de transcrição que regula vários genes envolvidos no metabolismo lipídico e glicídico. Níveis plasmáticos de adiponectina (produto de secreção do tecido adiposo) são importantes preditores independentes do perfil lipídico em pacientes portadores de FCH, incluindo níveis altos de triglicérides (TG), baixos de HDL-C e quantidades maiores de partículas de LDL (low-density lipoproteins - lipoproteína de baixa densidade) pequenas e densas, independentemente da resistência à insulina e da obesidade (3).

FCH está associada ao aumento da produção hepática de VLDL (very low density lipoprotein lipoproteína de densidade muito baixa) e freqüentemente à diminuição do clearence de lipoproteínas ricas em TG (31). Estas alterações são acompanhadas de níveis elevados de apolipoproteína-B (apo-B) e de LDLs pequenas e densas.
As LDLs representam um espectro de partículas de diferentes tamanhos e de proporções de colesterol e TG; as partículas pequenas e mais densas apresentam menor proporção de colesterol e maior de TG, sua preponderância aumenta com elevação de TG (o nível em que se tornam mais freqüentes situa-se na faixa de $150 \mathrm{mg} / \mathrm{dL}$ ). As LDLs pequenas e densas, mais aterogênicas, apresentam menor afinidade pelo receptor de LDL e são mais susceptíveis à oxidação.

As LDLs e também outras partículas ricas em triglicérides, tais como as lipoproteínas de densidade muito baixa (VLDL) e lipoproteínas de densidadeintermediária (IDL), contêm apo-B-100. Cada partícula destas lipoproteínas contém uma molécula de apo-B-100; assim, os níveis de apo-B-100 retratam o número destas partículas aterogênicas. Estudos mostraram que a medida de apo-B é um melhor marcador de risco cardiovascular do que os níveis de LDL-C. Contudo, a medida de apo-B é dispendiosa e apresenta dificuldades técnicas, não sendo utilizada rotineiramente. Há uma grande correlação entre os níveis de apo-B e os níveis de não-HDL colesterol (colesterol total menos HDL colesterol). Este índice pode ser uma medida presumida da apo-B, especialmente útil em pacientes com níveis mais elevados de TG e que têm maior proporção de colesterol nas VLDL e IDL. Em pacientes com níveis de TG $\geq 200 \mathrm{mg} / \mathrm{dL}$, o ATP III propõe alvos para não HDL-colesterol $(30 \mathrm{mg}$ acima dos estabelecidos para LDL colesterol) (4).

Resistência à insulina é característica da $\mathrm{FCH}$, não completamente explicada pelo aumento do IMC, e está associada ao fenótipo da expressão lipídica (5). Vários estudos utilizando clamp euglicêmico hiperinsulinêmico mostraram que os pacientes com FCH são resistentes à insulina $(6)$.

FCH é caracterizada pela expressão de múltiplos fenótipos de lipoproteínas. Numa mesma família afetada, indivíduos podem apresentar aumento de colesterol total, aumento de TG ou combinação de ambos.

Resistência à insulina está associada a hipertrigliceridemia isolada ou combinada a hipercolesterolemia. Este fato poderia estar relacionado ao aumento de IMC; entretanto, mesmo após correção pelo IMC, persiste a relação entre aumento de TG e resistência à insulina.

Diminuição dos níveis de TG com fibratos não melhoram a sensibilidade à insulina medida com a técnica de clamp. Por outro lado, indivíduos portadores de hipercolesterolemia apresentam a sensibilidade à insulina similar a normolipidêmicos de IMC correspondente (7). 
Disbetalipoproteinemia (Tipo III) é uma doença rara $(0,5 \%$ das famílias com DAC prematura). Em indivíduos normais, apo-E se liga a receptores de LDL e de quilomicrons com alta afinidade, promovendo a metabolização de VLDL, remanescentes de VLDL e remanescentes de quilomicrons; indivíduos portadores de disbetalipoproteinemia são homozigotos para apo-E2, que apresenta menor ligação aos receptores levando ao acúmulo de lipoproteínas, especialmente beta VLDL, altamente aterogênica e que leva a aumentos de colesterol e TG (8).

\section{Niveis ideais de lípides}

O ATP estabeleceu o que são considerados níveis baixos de HDL-CAo longo dos anos, os níveis ideais de LDL-C necessários para otimizar a redução do risco cardiovascular foram modificados, diminuindo marcadamente. Na última década, o alvo de LDL-C para pacientes de alto risco caiu para igual ou menor a 100 $\mathrm{mg} / \mathrm{dL}$ no NCEP-ATP II (9) e NCEP-ATP III (10) (vide tabela para níveis alvo de LDL colesterol). No final de 2004, o NCEP-ATP III (11) publicou adendo criando uma categoria diferenciada de pacientes de "risco muito alto", que inclui indivíduos com DAC estabelecida associada a outros fatores de risco importantes como diabetes e tabagismo: para este grupo está indicado LDL-C $<70 \mathrm{mg} / \mathrm{dl}$ e não HDL$\mathrm{C}<100 \mathrm{mg} / \mathrm{dL}$. A European Task Force on Prevention of Cardiovascular Disease também diminuiu os alvos de LDL-C na última década (12).

Estudos, como o HPS (13) e PROVE-IT (14), avaliaram a influencia de diminuição de LDL em níveis $<100 \mathrm{mg} / \mathrm{dL}$ como fator redutor de risco cardiovascular.

No estudo HPS (13), pacientes cujos níveis de LDL-C médios basais eram de $116 \mathrm{mg} / \mathrm{dL}$, e mesmo o subgrupo com concentrações de LDL-C < $100 \mathrm{mg} / \mathrm{dL}$, apresentaram redução significativa do risco quando a terapia com estatina foi introduzida. No estudo PROVE IT-(14), terapia intensiva redutora LDL-C com doses elevadas de atorvastatina $(80 \mathrm{mg} / \mathrm{dia})$ reduziu eventos cardiovasculares após 2 anos quando comparado com grupo controle tratado com pravastatina $40 \mathrm{mg} /$ dia. A pravastatina $40 \mathrm{mg} /$ dia reduziu o LDL-C de uma mediana de $106 \mathrm{mg} / \mathrm{dL}$ para $95 \mathrm{mg} / \mathrm{dL}$ (de acordo com alvo do ATP III < de $100 \mathrm{mg} / \mathrm{dL}$ ), e atorvastatina 80 $\mathrm{mg} /$ dia diminuiu LDL-C a uma mediana de 62 $\mathrm{mg} / \mathrm{dL}$.

Ambos os estudos, HPS e PROVE-IT, mostraram benefícios adicionais da redução substancial dos níveis de LDL-C abaixo de $100 \mathrm{mg} / \mathrm{dL}$.
Se extrapolarmos os resultados do HPS, uma redução de 30\% no LDL-C a partir de níveis de 100 $\mathrm{mg} / \mathrm{dL}$ produziria diminuição de $20 \%$ a $30 \%$ no risco relativo para DAC. Entretanto, o HPS e PROVE-IT não podem ser tomados como palavra final.

Existe um grande debate se o enfoque terapêutico deva ser exclusivamente centrado na redução dos níveis de LDL-C ou também ter como alvo o controle dos níveis de HDL-C e TG, particularmente importante no grupo de pacientes portadores de síndrome metabólica que apresentam alta freqüência de hiperlipidemia combinada.

\section{A importância clínica de níveis séricos baixos de HDL-C}

Níveis baixos de HDL-C freqüentemente são pouco valorizados como fator de risco para a aterosclerose prematura, embora dados epidemiológicos comprovem que HDL baixo está associado com DAC (15-17). Níveis séricos baixos de HDL-C são encontrados especialmente em pacientes obesos, e portadores de síndrome metabólica e diabetes tipo 2 .

O mecanismo primário da proteção cardiovascular das HDL parece estar relacionado ao aumento do transporte reverso do colesterol e a seus efeitos antiinflamatórios, antitrombóticos e antiproliferativos (18).

A resistência à insulina está também associada a níveis diminuídos do apolipoproteína A-l (apo-Al) e HDL-C (20); níveis elevados de ácidos graxos livres downregulate o transportador $\mathrm{ABCAl}$, envolvido no transporte reverso de colesterol. A resistência à insulina está associada a partículas menores e menos eficientes de HDL, bem como partículas maiores de VLDL. O tratamento farmacológico mais eficaz para HDL-C baixo é niacina.

É digno de nota que está aumentando a prevalência de HDL baixo na população, tendo sido registrada diminuição de $3,1 \mathrm{mg} / \mathrm{dL}$ na média de HDL-C entre 1981 e 1993, relacionada presumivelmente a obesidade e sedentarismo (19).

A primeira etapa para elevar os níveis de HDLC são as modificações de estilo de vida, mas o real papel do exercício tem sido superestimado e discute-se se, para baixos níveis de HDL, são realmente benéficos os exercícios intensivos. O exercício, entretanto, tem um bom efeito no HDL-C nos portadores de TG elevados (32).

\section{Medidas terapêuticas - dieta}

Dietas pobres em gorduras saturadas combinadas a exercícios físicos diminuem colesterol total, LDL-C e TG em 7-18\%, 7-15\% e 4-18\%, respectivamente, aumentando o HDL-C em 5 a 14\% (32). 
Embora as recomendações dietéticas sejam importantes, em geral não são suficientes para induzir uma diminuição eficaz do risco cardiovascular.

\section{As opções terapêuticas}

Embora as modificações de estilo de vida como exercício e perda de peso sejam importantes para indivíduos com dislipidemia combinada, estas intervenções em geral não são suficientes para se atingir os alvos estabelecidos no ATP III. A monoterapia com drogas hipolipemiantes pode provocar múltiplas modificações nos lípides, mas a combinação de drogas pode ser necessária para um manuseio mais abrangente nestes indivíduos.

\section{Estatinas}

Estatinas são inibidores estruturais da 3-hidroxi-3metilglutaril CoA redutase (HMG-CoA), enzima limitadora da biossíntese hepática de colesterol, resultando em upregulation dos receptores de LDL que levam à diminuição dos níveis de LDL-C. As estatinas compartilham uma estrutura similar que inibe a enzima e constituem a classe de medicamentos mais potente na redução dos níveis de LDL-C (21).

Existe discussão se os efeitos das estatinas seriam exclusivamente dependentes da diminuição de lípides ou em parte dependentes dos efeitos pleiotrópicos, e que compreenderiam vasodilatação, ação antitrombótica, antiinflamatória e antiproliferativa $(21,22)$.

Parece não haver diferenças entre as estatinas sobre os efeitos ditos pleiotrópicos, quer sejam elas derivadas de fungos (lovastatina, sinvastatina, e pravastatina) ou sintéticas (cerivastatina, fluvastatina, atorvastatina e rosuvastatina), hidrofilicas ou lipofílicas (21).

Redução de eventos cardiovasculares ocorre com todas as estatinas, dependendo o efeito da eficácia em modificar lipoproteínas. Também, terapias que não utilizavam estatinas, como fibratos e niacina, reduziram eventos cardiovasculares. Um dos estudos clínicos mais importantes que refuta o benefício especial das estatinas, além das mudanças no perfil de lípides do plasma, é o estudo POSCH (23), que demonstrou correlação entre o efeito do bypass ileal parcial, reduzindo LDL-C em 38\%, e a diminuição na freqüência de eventos cardiovasculares em 35\%. O efeito redutor de LDL-C decorrente deste tratamento cirúrgico foi quase idêntico ao das estatinas em reduzir LDL e diminuir eventos cardiovasculares.

Por outro lado, estudos recentes indicam que alguns dos efeitos pleiotrópicos das estatinas são independentes do colesterol e são responsáveis pela melhora da função endotelial, aumento da estabilidade de placa aterosclerótica, diminuição do estresse oxidativo e da inflamação, e ainda inibição da resposta trombogênica. Muitos destes efeitos pleiotrópicos parecem ser mediados pela inibição dos isoprenóides, que servem como ligantes lipídicos de moléculas sinalizadoras intracelulares (22).

$\mathrm{O}$ efeito predominante das estatinas é verificado sobre os níveis de LDL-C, mas reduzem também TG e aumentam modestamente o HDL-C. Embora a eficácia na redução do LDL-C varie entre estatinas, em média a redução do LDL-C varia entre $18 \%$ e $55 \%$, a redução de TG entre 7\% e 30\%, e o aumento de HDLC entre $5 \%$ e $15 \%$ (10).

Em uma análise conjunta de 7 estudos avaliando a eficácia das estatinas (em todos, pacientes com $\mathrm{TG} \geq 400 \mathrm{mg} / \mathrm{dL}$ foram excluídos), as concentrações de TG diminuíram proporcionalmente à redução do LDL-C quando os níveis de TG basais estavam abaixo de $250 \mathrm{mg} / \mathrm{dL} \mathrm{(10).}$

Estudos clínicos, apesar de não terem sido projetados para examinar a eficácia das estatinas na dislipidemia combinada, demonstraram redução na freqüência de eventos cardiovasculares em pacientes com TG e LDL-C elevados. No estudo de prevenção primária West of Scotland Coronary Prevention Study, a redução de eventos cardiovasculares com pravastatina foi de $29 \%$ em homens hipercolesterolêmicos com valores de TG abaixo da mediana de $148 \mathrm{mg} / \mathrm{dL}$, e de $32 \%$ naqueles com TG acima deste número (26).

Em análise post hoc do estudo Scandinavian Simvastatin Survival Study, em que todos os pacientes tinham LDL-C elevado, o grupo com TG no quartil mais elevado (> $159 \mathrm{mg} / \mathrm{dL}$ ) e HDL-C no quartil mais baixo $(<39 \mathrm{mg} / \mathrm{dL})$ teve maior redução em eventos cardiovasculares (52\%) com uso da sinvastatina (27).

\section{Diferenças farmacológicas}

Lovastatina, sinvastatina e atorvastatina são metabolizadas pelo sistema do citocromo (CYP) 450 3A4. Fluvastatina é metabolizada pela via $2 \mathrm{C} 9$ e a cerivastatina por ambas, 2C9 (ou 2C8) e 3A4. Pravastatina e rosuvastatina não são metabolizadas significativamente pela via da CYP (21). O uso concomitante de duas drogas metabolizadas pela CYP3A4 leva à competição pela via, resultando em concentrações aumentadas de ambas.

Além da ciclosporina, outros inibidores CYP3A4 que causam grande interação medicamentosa são: eritromicina, cetoconazole, nefazodona e inibidores de protease, havendo vários relatos de rabdomiólise quando lovastatina foi combinada com eritromicina (28). Quando macrolídeo é necessário, deve ser dada 
preferência a azitromicina, que não é metabolizada por esta via da CYP.

Os antidepressivos fluoxetina, fluvoxamina, nefazodona e sertralina inibem também CYP3A4 e devem ser usados cautelosamente com estatinas metabolizadas pela CYP3A4 (29). Alternativas para o tratamento da depressão sem interferência da CYP3A4 são a paroxetina e venlafaxina.

Inibidores de protease, além de induzir dislipidemias (indinavir, nelfinavir, ritonavir e saquinavir) inibem o metabolismo via CYP3A4, sendo requerida cautela com a co-adminstração com estatinas (30). Pravastatina, fluvastatina e atorvastatina em doses baixas podem ser utilizadas (33), e indinavir é, dentre os inibidores, o que menos afeta a CYP3A4.

Interação ocorre também entre as estatinas e os anticoagulantes cumarínicos, e a co-administração de estatinas a pacientes em uso varfarina pode causar um pequeno aumento no efeito do anticoagulante, sendo geralmente necessária redução na dose de varfarina. Casos de rabdomiólise foram relatados com a combinação do varfarina-estatina (30).

\section{Efeitos adversos das estatinas}

Aumento de enzimas hepáticas ocorre entre 0,5 a 2,5\% dos casos. Estas alterações são dose-dependentes e problemas graves são excepcionalmente raros, melhorando com redução da dose ou suspensão da medicação (34).

A miopatia tem uma freqüência geral baixa, acometendo aproximadamente $0,08 \%$ dos pacientes em uso de lovastatina e sinvastatina (35), podendo, entretanto, evoluir para rabdomiólise e insuficiência renal.

Algumas precauções são necessárias para se evitar miopatias associadas as estatinas (36):

a) avaliar a função tireoidiana antes de iniciar a terapia (dosagem de TSH); estatinas não devem ser administradas a pacientes com hipoptireoidismo;

b) considerar as interações medicamentosas das estatinas;

c) esclarecer pacientes no sentido de reconhecerem sintomas de miopatias;

d) maior cuidado nas seguintes condições: idosos (particularmente mulheres), portadores de disfunção renal ou hepática, diabéticos com evidências de esteatose hepática, hipotireidismo, usuários de drogas, cirurgia, trauma, isquemia-reperfusão, estados debilitantes, excessiva ingesta de álcool e exercícios intensos;

e) aconselhar os pacientes a suspenderem estatinas em caso de intercorrência clínica;

f) considerar suspensão quando for necessário uso de antibióticos macrolídeos; g) suspeitar de miopatia em caso de sensibilidade, fraqueza ou dor muscular generalizada ou câimbras noturnas;

h) avaliar CPK se houver sintomas de miopatias: se a CPK estiver até 5 vezes o limite superior da normalidade, repetir em 1 semana, se estiver acima de 5 vezes o limite superior da normalidade, descontinuar a medicação;

i) considerar as diferenças farmacocinéticas em pacientes que requeiram drogas que interfiram na CYP3A4;

j) não prescrever altas doses de estatinas em pacientes com insuficiência renal ou em combinação com fibratos e ciclosporina.

A freqüência de rabdomiólise parece diferir um pouco entre as estatinas, tendo sido retirada do mercado a cerivastatina devido à incidência aumentada destas complicações (37).

\section{Estatina e imunossupressores}

$\mathrm{O}$ risco de miopatia é muito aumentado com o uso de ciclosporina; $8 \%$ dos 601 casos de rabdomiólise induzidos por estatinas relatados pelo FDA entre março 1997 e novembro 2000 foram associados ao uso de ciclosporina $(38)$.

Nenhuma elevação de CPK, miosite ou de rabdomiólise foi verificada em estudo randomizado com a pravastatina em transplantados (39). Num período de observação de 8 anos com sinvastatina, elevações do nível de CPK acima de $100 \mathrm{U} / \mathrm{L}$ foram observadas em 22 dos 72 pacientes (todas reversíveis e após a atividade muscular), mas nenhum caso do rabdomiólise foi observado (40). Fluvastatina foi estudada em aproximadamente 1.000 pacientes póstransplante renal (Estudo ALERT) (41) em uso concomitante com ciclosporina, acompanhados durante 5 a 6 anos, não havendo casos de miosite significante.

\section{Drogas que interferem na absorção de colesterol}

\section{Resinas seqüestradoras de ácidos biliares}

São substâncias que se ligam a ácidos biliares formando complexos, sendo eliminados pelas fezes, impedindo a recirculação destas substâncias. Para compensar essas perdas, o fígado aumenta a conversão de colesterol a ácidos biliares, reduzindo a colesterolemia. Em geral, provocam pequeno aumento dos TG, sendo pouco adequados para pacientes com triglicérides aumentados. Constituem, geralmente, alternativa para pacientes intolerantes a estatinas; podem ser associados a estatinas para acentuar a redução de LDL. Interferem, ainda, na absorção de vários medicamentos $\mathrm{e}$ 
provocam freqüentemente sintomas digestivos, especialmente constipação.

Recentemente o colesevelam foi introduzido no mercado, minimizando os efeitos colaterais digestivos e interferência medicamentosa (21).

As resinas seqüestradoras de ácidos biliares diminuem LDL-C em 15 a 25\%, podendo aumentar TG em até $20 \%$ (42).

\section{Produtos disponíveis no mercado norte americano:}

a) Colestiramina (Questran), 4-16 g/dia (presente no mercado brasileiro);

b) Colestipol (Colestid), 5-20 g/dia; e

c) Colesevelam (WelChol), tabletes c/ $625 \mathrm{mg} ; 6-7$ $\mathrm{tab} / \mathrm{dia}$.

\section{Efeitos colaterais:}

Gastrointestinais: constipação, dor abdominal, flatulência. Ausência de toxicidade sistêmica. Inadequado em pacientes com níveis elevados de TG e dislipidemia do tipo III.

\section{Interações medicamentosas:}

Ligação com outras drogas com carga negativa. Impede a absorção de drogas e vitaminas lipossolúveis. Em geral utilizar outras drogas 1 hora antes ou 4-6 horas após

\section{Ezetimiba}

Inibe seletivamente a absorção intestinal de colesterol, levando a um menor aporte de colesterol ao fígado, aumentando conseqüentemente a expressão hepática de receptores de LDL. Ezetimiba e seu glicuronídeo (metabólito ativo) têm recirculação entero-hepática diminuindo a exposição sistêmica à droga. Geralmente é muito bem tolerada; aumento de enzimas hepáticas e distúrbios digestivos (especialmente diarréia) são pouco freqüentes (43).

O uso isolado provoca diminuição nos níveis de LDL-C em torno de 15-20\%, com efeitos positivos sobre os níveis de TG e HDL-C. É utilizado em dose fixa diária de $10 \mathrm{mg}$.

Quando associado as estatinas, reduz em média os níveis de LDL-C em 18\%, além da queda observada com a estatina (44).

Embora as estatinas sejam seguras até doses de $40 \mathrm{mg} /$ dia, aumentando-se a dose de 40 a $80 \mathrm{mg} /$ dia há um aumento significativo de alterações da função hepática e miopatia $(0,07 \%$ a $0,40 \%$ para a sinvastatina), embora a redução adicional de LDL-C seja de 5 a $6 \%$. Para pacientes que apresentam risco elevado de efeitos colaterais com estatinas, a adição de ezetimiba $10 \mathrm{mg}$ a $10 \mathrm{mg}$ de estatina apresentaria eficácia comparável à observada com $80 \mathrm{mg}$ da estatina e maior segurança.

Ezetimiba pode ser associada a fibratos, com excelente tolerância, para melhorar os efeitos na redução dos níveis de colesterol total, LDL-C e apo-B.

Dentre as drogas que diminuem a absorção de colesterol, a ezetimiba apresenta vantagens sobre as drogas seqüestradoras de ácidos biliares por sua segurança e tolerabilidade, constituindo importante ferramenta no controle dos níveis de colesterol.

\section{Controle dos níveis de HDL e TG elevados}

Tabela 1. Efeitos de drogas nos níveis de triglicérides e HDLC (42).

\begin{tabular}{lcc}
\hline & HDL-C & Triglicérides \\
\hline Ácido Nicotínico & $\uparrow 10-15 \%$ & $\downarrow 20-50 \%$ \\
Fibratos & $\uparrow 10-25 \%$ & $\downarrow 20-50 \%$ \\
Estatinas & $\uparrow 5-10 \%$ & $\downarrow 10-25 \%$ \\
Resinas sequestradoras sais biliares & $\uparrow 3-5 \%$ & $\uparrow 0-20 \%$ \\
Fish oils ácidos graxos $\gamma 3$ & - & $\downarrow 30-40 \%$ \\
\hline
\end{tabular}

Embora estatinas tenham efeitos sobre os TG, ele é pequeno comparado aos fibratos e ácido nicotínico. Ácidos graxos $\gamma 3$ também reduzem os TG, porem são necessárias doses muito elevadas.

\section{Fibratos}

\section{Efeitos sobre lípides}

Os fibratos diminuem os níveis de TG de 25-50\%, aumentam HDL-C em 15-25\%, sendo este efeito mais pronunciado em pacientes com hipertrigliceridemia (10). O efeito sobre os níveis de LDL-C é variável; reduzem os níveis em pacientes com TG baixos, mas os níveis podem até se elevar em pacientes com níveis altos de TG (21). Fibratos também diminuem significativamente os níveis de lipoproteínas remanescentes, altamente aterogênicas, sendo mais eficientes que as estatinas (49).

Fibratos aumentam os níveis séricos do HDL-C estimulando a expressão hepática das apoliproteínas apo-AI e apo-AII $(50,51)$. Múltiplos estudos demonstraram redução do risco cardiovascular associada ao aumento dos níveis séricos de apo A-I (52). Fibratos aumentam a expressão de ABCAl em macrófagos e o transporte reverso de colesterol (53).

Fibratos estimulam o metabolismo de triglicérides por diversas vias. A apolipoproteína C-III (apoCIII) é um inibidor potente da lipase lipoprotéica; fibratos inibem a expressão da apo-CIII estimulando 
expressão da lipase lipoprotéica, que auxilia a metabolização dos triglicérides das VLDL e quilomicrons (54). Quando ácidos graxos livres são liberados dos triglicérides, os fibratos facilitam sua metabolização upregulating a expressão da carnitina palmitoiltransferase I na membrana interna mitocondrial e o mecanismo enzimático necessário para b-oxidação dos ácidos graxos.

A inflamação desempenha um papel chave na etiologia da aterosclerose. Interleucinas, citocinas e enzimas modificadoras de matrizes intercelulares interagem com diversos tipos celulares promovendo alterações que levam à formação de células espumosas, estrias gordurosas e da placa ateromatosa (55).

Fibratos são agonistas do $\operatorname{PPAR} \alpha$, um importante mediador da inflamação e do status redox celular. A via nuclear do fator $\kappa \mathrm{B}(\mathrm{NF \kappa B})$ regula a expressão de moléculas da adesão, de interleucina 6 , de ciclooxigenase 2 e da endotelina 1. PPAR $\alpha$ inibe NFKB

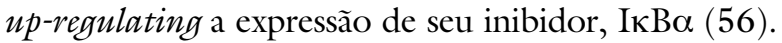

PCR (proteína C reativa) é um modulador importante e marcador da doença aterosclerótica; terapia agonista PPAR $\alpha$ com fibrato diminui os níveis séricos de PCR (57). PPAR $\alpha$ modula também a capacidade das células de inativar espécies reativas de oxigênio.

\section{Fibratos - Preparações e doses diárias:}

a) Bezafibrato - 400 a $600 \mathrm{mg} / \mathrm{dia}$;

b) Fenofibrato - $200 \mathrm{mg} / \mathrm{dia}$;

c) Ciprofibrato - $100 \mathrm{mg} / \mathrm{dia}$;

d) Gemfibrozil - 600 a $1200 \mathrm{mg} / \mathrm{dia}$

\section{Efeitos Colaterais:}

Distúrbios gastrointestinais (6\%), colelitíase, miosite e aumento de enzimas hepáticas.

\section{Contra-indicações:}

Disfunção renal ou hepática e colecistopatia

\section{Ensaios clínicos com fibratos}

Estudos demonstram a importância de se aumentar HDL sérico e de se reduzir os TG na prevenção primária e secundária de eventos cardiovasculares e cerebrovasculares agudos.

O Helsinki Heart Study (58) é um estudo de prevenção primária que envolveu 4.081 homens apresentando níveis de não-HDL-C $\geq 200 \mathrm{mg} / \mathrm{dL}$, e que usaram gemfibrozil (1200 mg/dia) ou placebo. O grupo tratado apresentou uma redução total de $34 \%$ $(\mathrm{p}<0,02)$ no risco para primeiro evento DAC. Entre os homens que apresentavam triglicérides > 200 $\mathrm{mg} / \mathrm{dL}$ e $\mathrm{HDL}<42 \mathrm{mg} / \mathrm{dL}$, o risco diminuiu quase

Tabela 2. Estratificação de risco cardiovascular estabelecido pelo National Cholesterol Expert Panel (NCEP) segundo os níveis de LDL-C.

\begin{tabular}{|c|c|c|c|}
\hline \multirow{2}{*}{$\begin{array}{l}\text { Parâmetros do NCEP } \\
\text { Categoria de RISCO }\end{array}$} & \multicolumn{3}{|c|}{ Niveis de LDL-C em mg/dL (1 1) } \\
\hline & Objetivos & $\begin{array}{l}\text { Iniciar mudanças } \\
\text { de hábitos }\end{array}$ & $\begin{array}{l}\text { Indicação de } \\
\text { drogas }\end{array}$ \\
\hline 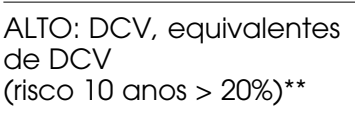 & $<100$ & $\begin{array}{l}\quad \geq 100 \\
\text { alvo opcional } \\
<70 \mathrm{mg}\end{array}$ & $\begin{array}{c}\geq 10 \\
(<100-\text { considerar } \\
\text { uso de drogas })\end{array}$ \\
\hline $\begin{array}{l}\text { MODERADAMENTE ALTO } \\
2 \text { ou mais FR* } \\
\text { (risco } 10 \text { anos - } 10 \text { a } 20 \% \text { ) }\end{array}$ & $<130$ & $\geq 130$ & $\begin{array}{l}\geq 130 \\
\text { (100-129 considerar } \\
\text { uso de drogas) }\end{array}$ \\
\hline 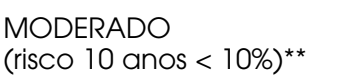 & $<130$ & $\geq 130$ & $\geq 160$ \\
\hline BAIXO 0-1 FR* & $<160$ & $\geq 160$ & $\begin{array}{l}\geq 190 \\
\text { (160-189: uso de } \\
\text { drogas opcional) }\end{array}$ \\
\hline
\end{tabular}

Risco muito alto - DCV associada a diabetes mellitus, tabagismo ou doença metabólica (TG $\uparrow$ e HDL $\downarrow$ )

Equivalentes de DCV - diabetes mellitus, doença arterial periférica, aneurisma de aorta abdominal e doença arterial carotídea sintomática

${ }^{*}$ FR= fatores de Risco: tabagismo; pressão arterial $\geq 140 / 90 \mathrm{mmHg}$ ou terapêutica antihipertensiva; $\mathrm{HDL}-\mathrm{C}<40$ mg/dL; História familiar de doença coronariana prematura em parentes do 10 grau: (homens < 55 anos mulheres < 65 anos); ldade: homens $\geq 45$ anos e mulheres $\geq 55$ anos

** Tabela de risco estimado de doença cardiovascular segundo Framingham (10). 
72\%. Em 135 pacientes diabéticos que participaram do estudo, a terapia com gemfibrozil reduziu o risco de eventos cardiovasculares agudos em 65\%, embora este dado não fosse estatisticamente significativo (provavelmente devido ao baixo número de eventos).

No estudo VA-HIT (Veterans Affairs HighDensity Lipoprotein Intervention Trial) (59), homens com DAC e média de LDL de $111 \mathrm{mg} / \mathrm{dL}$, médias de HDL de $31 \mathrm{mg} / \mathrm{dL}$, e médias de triglicérides de 161 $\mathrm{mg} / \mathrm{dL}$ receberam gemfibrozil (1200 mg/dia) ou placebo, sendo acompanhados por um período de 5 anos. No grupo tratado, foi verificada uma elevação de 6\% nos níveis de HDL-C, sem alterações no LDL-C, e uma diminuição de $31 \%$ nos triglicérides. Os pacientes tratados com gemfibrozil tiveram uma redução de $22 \%$ $(\mathrm{p}=0,006)$ na mortalidade total e nos infartos do miocárdio não fatais.

O tratamento com gemfibrozil também reduziu os riscos de acidentes vasculares cerebrais e ataques isquêmicos transitórios em $31 \%(\mathrm{p}=0,036)$ e $59 \%(\mathrm{p}<$ $0,001)$, respectivamente; a necessidade de endarterectomia carotídea foi de $65 \%(\mathrm{p}<0,001)$ menor. A redução da freqüência de eventos cerebrovasculares com terapia do gemfibrozil foi aparente em 6 a 12 meses. Os pacientes diabéticos que participaram do VA-HIT também se beneficiaram com o uso do gemfibrozil, com reduções de $32 \%(\mathrm{p}=0,004)$ em eventos combinados, $41 \%(\mathrm{p}=0,02)$ na morte por DAC e de $40 \%$ por acidente vascular cerebral $(\mathrm{p}=0,046)(59)$.

$O$ benefício da terapia do gemfibrozil foi proporcional à resistência à insulina. VA-HIT foi o primeiro estudo a demonstrar redução de eventos cardiovasculares e cerebrovasculares por intervenção com medicação hipolipemiante, independente de alteração do LDL-C. A maior parte do benefício da terapia com fibrato neste estudo foi atribuída à elevação de HDL e aos efeitos pleiotrópicos do gemfibrozil.

O Bezafibrate Infarction Prevention (BIP) (60), estudo de prevenção secundária, comparou uso de bezafibrato $(400 \mathrm{mg} / \mathrm{d})$ ao placebo em 3.122 homens

Tabela 3. Efeito de hipolipemiantes nos níveis de LDL-C (adaptado da ref. 10).

\begin{tabular}{|c|c|}
\hline \multicolumn{2}{|c|}{ Dose dependente } \\
\hline Estatinas & $\downarrow 20-50 \%$ \\
\hline Resinas Seq. de Sais Biliares & $\downarrow 15-25 \%$ \\
\hline Ácido Nicotínico & $\downarrow 15-30 \%$ \\
\hline Ezetimiba & $\downarrow 20 \%$ \\
\hline \multicolumn{2}{|c|}{ Dose Fixa } \\
\hline Gemfibrozil & $\downarrow 10-15 \%$ \\
\hline Fenofibrato & $\downarrow 10-25 \%$ \\
\hline Ciprofibrato & $\downarrow 10-25 \%$ \\
\hline
\end{tabular}

e mulheres com DAC documentada. Os pacientes foram seguidos, em média, por 6,2 anos; os eventos primários avaliados foram infarto agudo do miocárdio fatal ou não fatal e morte súbita. Os parâmetros lipídicos médios do estudo eram: LDL-C $148 \mathrm{mg} / \mathrm{dL}$, HDL-C 34,6 mg/dL e triglicérides $145 \mathrm{mg} / \mathrm{dL}$. Os pacientes que utilizaram bezafibrato apresentaram uma redução de $5 \%$ nos níveis de LDL-C, um aumento de $12 \%$ nos de HDL e uma diminuição de $22 \%$ nos TG.

A terapia com bezafibrato foi segura, não havendo diferenças quanto à incidência de mialgia, hepatotoxicidade e câncer entre os grupos estudados. $\mathrm{O}$ bezafibrato reduziu o risco de eventos primários em somente $7,3 \%(p=0,24)$, não sendo significativo. As reduções em eventos secundários, como angina instável, necessidade de revascularização, angioplastia coronariana ou cirurgia para revascularização miocárdica, mortalidade cardíaca e acidente vascular cerebral isquêmico, também não apresentaram diferenças estatisticamente significativas. Entretanto, em uma análise post hoc, em pacientes com níveis de triglicérides basais acima de $200 \mathrm{mg} / \mathrm{dL}$ e $\mathrm{HDL} \leq 35 \mathrm{mg} / \mathrm{dL}$, a terapia com bezafibrato reduziu a freqüência de eventos em $41 \%(p=0,02)(60)$.

As disparidades nos resultados entre o BIP o VA-HIT podem ser atribuídas aos seguintes fatos: $O$ nível basal do LDL nos estudos (111 mg/dL em VAHIT contra $148 \mathrm{mg} / \mathrm{dL}$ em BIP) pode ter tido papel significativo; o alvo do NCEP para LDL-C nos pacientes com DAC é < $100 \mathrm{mg} / \mathrm{dL}$; no VA-HIT, os pacientes estavam bem mais perto deste objetivo de LDL-C do que os do estudo BIP. Um outro ponto importante é que $15 \%$ do grupo do placebo no estudo BIP usou drogas redutoras de LDL-C, afetando possivelmente os resultados.

Vários estudos angiográficos [BECAIT (61), LOCAT (62), DAIS (63)] avaliaram terapia com fibrato, demonstrando que estas drogas reduzem as taxas da progressão da placa ateromatosa avaliada por estudos angiográficos.

Estudos relativamente pequenos e de curto prazo demonstraram que estatina e fibratos em combinação podem fornecer reduções aditivas no níveis séricos de LDL, triglicérides, e aumentando em HDL. O estudo SAFARI mostrou que associação de $20 \mathrm{mg}$ de sinvastatina e 160 de fenofibrato reduziu os níveis de LDL e TG em 31,2 e 49,1\%, respectivamente, e elevou HDL em 18,6\% (64).

No início da terapia, um paciente com hipertrigliceridemia pode ter níveis normais ou baixos de LDL-C; contudo, como os fibratos estimulam a con- 
versão de VLDL em LDL, à medida que as concentrações de triglicérides caem, os níveis de LDL-C podem subir, algumas vezes substancialmente (2l), e, nesta situação, a adição de estatina ou de algum inibidor da absorção de colesterol pode ser necessária.

Além disso, ao contrário do gemfibrozil, o fenofibrato pode apresentar capacidade adicional em reduzir níveis séricos de LDL.

A monoterapia com fibratos parece levar a um risco independente de miopatia maior que o verificado com estatinas em monoterapia. Uma análise dos dados de 17.219 pacientes de clínicas médicas no Reino Unido revelou que os usuários de fibratos tinham a probabilidade 5,5 vezes maior de desenvolver miopatia do que usuários de estatinas (65).

\section{Fibratos e função renal}

Broeders e cols. (66) avaliaram 27 pacientes que desenvolveram disfunção renal em uso de fibratos ( 25 fenofibrato, 1 bezafibrato, e 1 ciprofibrato), sendo 19/27 transplantados. Nestes pacientes, foi verificado aumento de $40 \%$ nos níveis de creatinina e 36\% nos níveis de uréia. A função renal retornou à linha de base em 18/24 pacientes após a suspensão da droga. Entretanto, seis pacientes, todos receptores de transplante, permaneceram com aumento da creatinina plasmática. A incidência de disfunção renal induzida pelo fibrato na série de pacientes transplantados renais foi de $60 \%$.

Aumento de creatinina foi relatado em diversos estudos que utilizaram fibratos, fenofibrato $(n=7)$ e bezafibrato $(n=8)$ (variação de $8-18 \%$ e $8-40 \%$, respectivamente), e em três de quatro estudos com ciprofibrato (variação de 6-16\%). Nenhum caso de comprometimento renal foi descrito em relatos de terapia do gemfibrozil. Assim, a terapia com fenofibrato, bezafi- brato e ciprofibrato pode induzir disfunção renal, enquanto o gemfibrozil parece ser isento deste efeito colateral (66).

Por outro lado, Hottelart e cols. (67) demonstraram que o aumento na creatininemia induzida pelo fenofibrato em pacientes renais crônicos não reflete insuficiência na função renal ou na secreção tubular de creatinina, ou interferência nas dosagens laboratoriais. $O$ aumento na produção de creatinina também não pode ser explicado por lise acelerada das células musculares. Os autores propuseram que o fenofibrato aumente a produção de creatinina (65).

\section{Interação estatina-fibrato}

Embora a monoterapia favoreça vários parâmetros lipídicos, a terapia combinada é freqüentemente necessária para se alcançar os objetivos mais estritos orientados pelos painéis especializados, principalmente em pacientes de alto risco e portadores de dislipidemia combinada e síndrome metabólica.

Há freqüentemente relutância em se prescrever concomitantemente estatinas e fibratos por preocupação com a interação destas drogas, aumentando risco de miopatia e rabdomiólise, portanto uma análise mais precisa dos riscos e interações medicamentosas pode fornecer subsídios para uma decisão terapêutica mais técnica e segura.

Estudos recentes demonstram diferenças farmacológicas entre o gemfibrozil e o fenofibrato. Estas drogas apresentam diferentes vias de metabolização. Todas as estatinas são reconhecidas por transporters, que são polipeptídeos transportadores orgânicos aniônicos. A interação entre o gemfibrozil e estatinas poderia resultar da interação com os transporters hepáticos, levando à diminuição da captação pelo fígado (68).

Tabela 4. Alterações médias das lípides observadas com doses máxima de cada estatina (adaptado de Jones e cols. (24) e Winkler e cols. (25)).

\begin{tabular}{|c|c|c|c|c|c|}
\hline & Rosuvastatina & Atorvastatina & Sinvastatina & Pravastatina & Fluvastatina \\
\hline Dose Máxima (mg/dia) & 40 & 80 & 80 & 40 & 80 \\
\hline $\begin{array}{l}\text { Diminuição } \\
\text { Colesterol Total (\%) }\end{array}$ & $-40,2$ & $-38,9$ & $-32,9$ & $-21,5$ & -23 \\
\hline Diminuição LDL-C (\%) & $-55,0$ & $-51,1$ & $-45,8$ & $-29,7$ & -29 \\
\hline Diminuição TG (\%) & $-26,1$ & $-28,2$ & $-18,2$ & $-13,2$ & -18 \\
\hline Aumento HDL-C (\%) & $+9,6$ & $+2,1$ & $+6,8$ & $+5,6$ & +3 \\
\hline Metabolização - CYP & P450-2C9 & $P 450-3^{A} 4$ & $\mathrm{P} 450-3^{\mathrm{A}} 4$ & não definida & P450-2C9 \\
\hline
\end{tabular}

Equivalência das Estatinas: doses que provocam reducão de $30-35 \%$ nos níveis de LDL-C

5 a 10 mg de Rosuvastatina; 10 mg de Atorvastatina; 20 mg Sinvastatina; 40 mg

Lovastatina/Pravastatina; 80 mg de Fluvastatina.

OBS: Dobrando-se a dose, há uma diminuição adicional de $\pm 6-8 \%$. 
Fenofibrato e o gemfibrozil diferem significativamente por serem metabolizados por diferentes enzimas hepáticas na sua glucoronidação (UGT 1A9 e 2 B7 para o fenofibrato e o UGT 1Al e 1 A3 para o gemfibrozil). Estas diferenças têm implicações clínicas significativas porque a maioria das estatinas é glucoronisada pela mesma família de enzimas que o gemfibrozil, competindo, desse modo, na conversão das estatinas da forma aberta para a forma lactona, que sofre metabolização pelo fígado e é posteriormente catalisada pelo citocromo. O fenofibrato é um inibidor leve de CYP2C9, enquanto o gemfibrozil é um potente inibidor da CYP2C9 e de CYP2C8 (68).

Estas diferenças parecem explicar a maior interação das estatinas com o gemfibrozil comparado com o fenofibrato. Gemfibrozil aumenta a área sob a curva da concentração de todas as estatinas (a atorvastatina não foi avaliada); enquanto o fenofibrato não aumenta significativamente a área sob a curva das concentrações de sinvastatina, cerivastatina, pravastatina ou rosuvastatina $(21)$.

Somente $2,3 \%$ (14/606 casos) do número total de relatos de rabdomiólise foram devidos à associação de estatina-fenofibrato, sendo a maior parte devida à combinação fenofibrato-cerivastatina $(88 \%$ - 533/ 606 casos). O número dos relatos de rabdomiólise por milhão de prescrições dispensadas era aproximadamente 33 vezes menor com fenofibrato do que com gemfibrozil quando estes foram usados em combinação com cerivastatina (69).

Quando fenofibrato foi combinado a outra estatina (exceto cerivastatina), houve um número bem menor de relatos de rabdomiólise. O número de relatos por milhão de prescrições dispensadas foi apro- ximadamente 15 vezes menor com fenofibrato do que para o gemfibrozil $(0,58$ vs. 8,6 relatos por milhão de prescrições) (68).

Análise da terapia combinada de estatinas e fibratos dos dados de 11 planos de saúde nos Estados Unidos, contabilizando hospitalizações entre $1 \underline{\text { o }}$ de janeiro de 1998 a 30 de junho de 2001, indicou que a cerivastatina combinada a um fibrato conferia um risco da hospitalização por rabdomiólise de aproximadamente 1 em 10 pacientes tratados por o ano. Nesta avaliação, 252.460 pacientes usaram drogas hipolipemiantes, havendo 24 casos de rabdomiólise, e somente 1 com fenofibrato (associado a atorvastatina 40 $\mathrm{mg} /$ dia) (70). Não há dados mais precisos sobre os riscos de rabdomiólise com o uso combinado de estatinas e ciprofibrato ou bezafibrato.

Revisão dos dados da Veterans Administration database avaliou 93.677 pacientes usando gemfibrozil combinado a estatina e 1.830 fenofibrato-estatina, sendo descritos 149 casos de rabdomiólise ou necrose tubular aguda. Nos 93.677 pacientes que fizeram uso gemfibrozil com estatina, a freqüência destas complicações foi de $0,16 \%$, não havendo nenhum caso de rabdomiólise ou necrose tubular agudo nos 1.830 pacientes que utilizaram a associação fenofibratoestatina (71).

No estudo Simvastatin plus Fenofibrate for Combined Hyperlipidemia, 411 pacientes receberam $20 \mathrm{mg}$ de sinvastatina em combinação com 160 $\mathrm{mg} /$ dia de fenofibrato por 12 semanas, não sendo descritos casos de miopatia (4 pacientes abandonaram o estudo por elevações de transaminases, 3 vezes acima dos limites da normalidade) (64).

Em uma revisão de 36 estudos clínicos publicados entre 1988 e 2000 (numerosos estudos foram

Tabela 5. Efeito do ezetimiba associado a estatinas e fenofibrato sobre as concentrações lípides (\%) (adaptado das ref. 45, 46, 47 e 48).

\begin{tabular}{lcccccc}
\hline Droga(s) & $\mathbf{N}^{\circ}$ de pacientes & Colesterol Total & LDL-C & HDL-C & TG & APOB \\
\hline Atorvastatina isolada & 248 & -32 & -44 & +4 & -25 & -36 \\
Atorvastatina+ezetimiba & 255 & -56 & -56 & +7 & -33 & -45 \\
Sinvastatina isolada & 263 & -26 & -36 & +7 & -17 & -30 \\
Sinvastatina+ezetimiba & 274 & -37 & -51 & +9 & -24 & -41 \\
Pravastatina isolada & 205 & -17 & -25 & +7 & -8 & -20 \\
Pravastatina+ezetimiba & 204 & -27 & -39 & +8 & -18 & -30 \\
Lovastatina isolada & 220 & -18 & -25 & +4 & -11 & -21 \\
Lovastatina+ezetimiba & 182 & -29 & -40 & +9 & -22 & -33 \\
Fenofibrato isolada & 179 & $-10,8$ & - & $+18,8$ & $-43,2$ & $-15,2$ \\
Fenofibrato+ezetimiba & 175 & $-22,4$ & - & +19 & -44 & $-26,1$ \\
\hline
\end{tabular}


de curta duração) envolvendo uma população total de 1.674 pacientes, a incidência total de miopatia foi de $0,12 \%$. Dos estudos revistos, 20 usaram gemfibrozil e 10 bezafibrato; nos 2 casos de miopatia descritos, o gemfibrozil era a medicação empregada (72).

Em relação ao bezafibrato, a combinação com fluvastatina não foi associada com elevações relevantes de CPK ou miopatia em 333 pacientes com hiperlipidemia combinada que foram seguidos durante 6 meses (73). Em outro estudo aberto de 12 meses de duração, miopatia foi descrita em 2 dos 148 pacientes com diabetes tipo 2 que receberam a combinação sinvastatina-bezafibrato (74).

Existem poucos dados sobre a associação estatina-ciprofibrato. Em 1998, um consenso americano indicou o uso clínico dos fibratos no tratamento do dislipidemia e DAC, confirmando os benefícios da terapia da estatina-fibrato nos pacientes com hiperlipidemia de tipo IIB (75); entretanto, advertiu que esta combinação não deve ser usada pelos seguintes grupos de pacientes: idosos (acima de 70 anos), renais crônicos, portadores de doenças músculo-esqueléticas, necessitando de múltiplas medicações (especialmente ciclosporina, tacrolimus, macrolídeos antifúngicos azóis). Pacientes com outras doenças graves, e que não tenham capacidade de compreender os riscos da terapia, também devem ser excluídos.

\section{Derivados do ácido nicotínico}

\section{Niacina}

A niacina modifica favoravelmente todas as principais frações lipídicas, sendo o único agente hipolipemiante que diminui a lipoproteína (a), e a droga mais eficaz na elevação dos níveis de HDL-C. Niacina pode reduzir os níveis de LDL-C em $5-25 \%$, e os de TG em 20-50\%, além de aumentar o HDL-C em $15-35 \%(21,42)$.

Niacina age inibindo o transporte dos ácidos graxos livres dos tecidos periféricos ao fígado; desta forma, reduz eficazmente a síntese hepática de TG, resultando na diminuição da produção de lipoproteínas contendo apo-B. Niacina diminui também o catabolismo das apo-AI (76).

Foi demonstrado que a niacina altera a distribuição das partículas de LDL aumentando a concentração de LDLs grandes e flutuantes, diminuindo a concentração das LDLs pequenas e densas, beneficiando o perfil lipídico.

\section{Produtos disponíveis mercado americano - dose diária:}

Liberação imediata - 2 a 4g/dia; Liberação prolongada (Niaspan) - 1 a $2 \mathrm{~g} /$ dia e OTC, liberação lenta $-<2$ $\mathrm{g} /$ dia.

Niacina é disponível nos Estados Unidos em 3 formulações. Duas disponíveis como preparações de venda livre, e incluem a niacina cristalina (de liberação imediata) e ao Slo-Niacina (Upsher-Smith, Minneapolis, $\mathrm{MN})(2 \mathrm{l})$.

No mercado brasileiro, duas formulações de niacina de liberação prolongada estão sendo comercializadas, embora não sejam exatamente iguais ao Niaspan.

\section{Efeitos adversos}

O uso da niacina pode se associar à doença péptica, náusea, sensação de má digestão, prurido e acantose nigricans. O problema mais freqüiente e limitador do uso da niacina é o flushing (enrubescimento) cutâneo. Outros efeitos adversos descritos incluem: cefaléia, efeitos gastrointestinais (principalmente na formula de liberação lenta) hepatoxicidade, ativação de úlcera péptica, hiperglicemia e redução da sensibilidade à insulina e aumento de ácido úrico. Niacina em uso isolado não está associada a miopatia.

\section{Contra-indicações}

Hepatopatias ou alterações nas provas de função hepática, e úlcera péptica.

Flushing decorre da vasodilatação dérmica mediada por prostaglandinas. Aspirina e outros salicilatos são inibidores potenciais da ciclooxigenase e da biossíntese de prostaglandinas, estes agentes ou agentes antiinflamatórios não hormonais podem ser usados para abrandar o flushing, que pode ocorrer em até $67 \%$ dos pacientes (21). A "Slo-Niacin" foi desenvolvida para retardar a absorção de niacina e diminuir o risco de flushing. Tanto a niacina cristalina quanto a Slo-Niacin estão associadas com o risco significativo de hepatotoxicidade, especialmente quando são usadas doses acima de $2 \mathrm{~g} /$ dia. Niaspan (Kos, Miami, FL) é uma formulação de liberação prolongada que permite a absorção da niacina em aproximadamente 8 horas, apresentando melhor tolerância e menor risco de hepatotoxicidade e miopatia $(77,78)$.

Niaspan pode aumentar as concentrações séricas de ácido úrico, possivelmente bloqueando a secreção tubular renal. Em alguns pacientes, a hiperuricemia pode exacerbar o risco de crises de gota $(77,78)$.

Niaspan pode também reduzir níveis do fósforo sérico em $9 \%$ e diminuir a contagem de plaquetas em $10-15 \%$ (78). 
Niaspan deve ser utilizado ao deitar, pois, desta forma, parece melhorar a tolerância em relação ao flushing. Também, durante a noite há aumento da lipólise do tecido adiposo, quando os triglicérides séricos atingem seus maiores valores do ciclo de 24 horas. Foi demonstrado que a niacina inibe a elevação noturna de triglicérides séricos, fato que pode resultar em benefícios adicionais no risco cardiovascular (79).

Monoterapia com niacina não parece estar associada com miopatia. Foram relatados casos de rabdomiólise quando niacina foi associada a lovastatina, pravastatina e sinvastatina, mas não com atorvastatina, fluvastatina ou rosuvastatina (21).

O Coronary Drug Project (80) foi o primeiro estudo a demonstrar que a melhora do padrão lipídico ajuda a prevenir doença cardiovascular, reduzindo mortalidade. Após 6 anos, a monoterapia com niacina reduziu a freqüência de infarto agudo do miocárdio não fatal em $27 \%$. Este estudo avaliou pacientes com infarto do miocárdio prévio, submetidos a terapia com niacina ( $3 \mathrm{~g} / \mathrm{dia})$; o colesterol total diminuiu em $10 \%$ e TG em 26\% comparado ao grupo placebo.

Niacina de liberação prolongada (Niaspan, na dose de 375-2000 mg/d) usada em monoterapia na dose de $2000 \mathrm{mg} /$ dia diminuiu LDL em 17\%, triglicérides em 35\%, e $\operatorname{Lp}($ a) em 24\%, aumentando ainda o HDL em 26\%. Estas alterações nas concentrações de lipoproteínas foram estáveis em 2 anos de seguimento (81).

O estudo Stockholm Ischemic Heart Disease Secondary Prevention Study demonstrou que o tratamento combinado da niacina e clofibrato diminuiu a mortalidade por doença isquêmica cardíaca (36\%) e total $(26 \%)$ em comparação com o placebo $(83)$.

Estudos angiográficos randomizados, controlados por placebo, demonstraram que a niacina, isoladamente ou em combinação com outras drogas hipolipemiantes, desaceleram a progressão da doença cardiovascular avaliada por angiografia. O estudo FATS (Familial Atherosclerosis Treatment Study), onde a niacina foi combinada ao colestipol, além dos efeitos na progressão da lesão, verificou diminuição de $73 \%$ na freqüência de eventos coronarianos agudos $(85)$.

O estudo HATS (HDL-Atherosclerosis Treatment Study) (86) avaliou a eficácia da terapia combinada em 160 homens e mulheres com DAC, HDL baixo (< $35 \mathrm{mg} / \mathrm{dL}$ nos homens, $<40 \mathrm{mg} / \mathrm{dL}$ nas mulheres) com seguimento de 3 anos Foi avaliada a associação sinvastatina ( 10 a $20 \mathrm{mg} /$ dia) e niacina (2000 a $4000 \mathrm{mg} /$ dia) versus placebo. Além dos efeitos sobre a progressão da lesão, a combinação niacina-sinvastatina diminuiu os óbitos relacionados a
DAC, IAM e acidente vascular cerebral, e a necessidade de revascularização em 90\% $(p=0,03)$. A coadministração de antioxidantes em combinação com niacina-sinvastatina suprimiu os benefícios da terapia, sendo a redução de eventos de somente $60 \%(\mathrm{p}=0,02)$.

A combinação de niacina de liberação imediata e gemfibrozil pode aumentar HDL em até 45\% (84).

\section{Niacina e diabetes mellitus}

Através de mecanismos ainda não esclarecidos, a niacina pode induzir elevações suaves na glicemia. Por esta razão, muitos clínicos ficam hesitantes em usar a niacina em pacientes com diabetes mellitus ou intolerância à glicose (IGT). No estudo ADVENT (Assessment of Diabetes Control and Evaluation of the Efficacy of Niaspan Trial), foi avaliado o efeito de duas doses de Niaspan versus placebo no controle glicêmico em 148 pacientes com diabetes mellitus tipo 2 mellitus, seguidos por 4 meses (81).

Os valores de hemoglobina glicosilada basais e após 4 meses de terapia eram os seguintes, respectivamente: 1) Niaspan $1000 \mathrm{mg} / \mathrm{dia}$ (placebo - 7,13\% e $7,11 \%$; Niaspan $-7,28 \%$ e 7,35\% $[\mathrm{p}=0,16$ contra o placebo]); 2) Niaspan $1500 \mathrm{mg} / \mathrm{d}(7,2 \%$ e $7,5 \%$ [p= 0,48 contra o placebo)]. Em pacientes diabéticos, estas pequenas mudanças no controle glicêmico podem ser corrigidas ajustando-se a dose de insulina ou medicação hipoglicemiante. Em pacientes intolerantes à glicose (IGT) ou que apresentem glicemia de jejum entre 100-125 mg/dL, a terapia com doses elevadas de niacina pode prejudicar o metabolismo glicídico o suficiente para permitir o diagnóstico de diabetes mellitus (glicemia de jejum > $126 \mathrm{mg} / \mathrm{dL}$ ). Conseqüentemente, pacientes intolerantes à glicose devem ser cuidadosamente monitorados caso utilizem niacina.

Os estudos ADMIT (87) e de ADVENT (81) demonstraram, também, que a terapia com niacina altera níveis séricos de inúmeros fatores de risco cardiovasculares emergentes. O estudo ADVENT mostrou uma tendência à redução dos níveis de PCR (placebo 2\%; Niaspan $1000 \mathrm{mg} /$ dia 11\% e Niaspan $1500 \mathrm{mg} /$ dia $20 \%$ ).

Monoterapia com ácido nicotínico (niacina) tem sido menos freqüentemente utilizada para diminuir os níveis de LDL-C, porque não é bem tolerada nas doses elevadas requeridas em monoterapia. Entretanto, tem sido cada vez mais utilizada em associação com estatinas, acentuando as reduções de LDL-C e triglicérides e aumentando HDL-C.

Como em monoterapia, com um ou outro agente, os pacientes submetidos à terapia combinada estatina-niacina devem ter as transaminases monito- 
radas e devem ser advertidos para relatar todos os sintomas que sugiram miopatia.

Face ao elevado risco cardiovascular associado ao diabetes e síndrome metabólica, o Consenso Europeu (82) indica o manuseio agressivo de fatores de risco como prioridade clínica. As regras atuais preconizam inicialmente medidas higiênicas e dietéticas e enfatizam o uso de estatinas. Embora estas sejam efetivas em diminuir o risco cardiovascular em pacientes diabéticos (não há muitas evidências em portadores de síndrome metabólica), persiste neste grupo um alto risco cardiovascular residual. Claramente, há necessidade de corrigir os outros distúrbios lipídicos como HDL-C baixo, TG elevado, LDLs pequenas e densas, característicos da dislipidemia aterogênica. Associações medicamentosas das estatinas com ácido nicotínico ou fibratos são as alternativas disponíveis.

Estudos recentes mostraram que ácido nicotínico pode ser usado em diabéticos (inclusive tendo sido autorizado pela ADA); o painel Europeu indica a associação estatina-niacina $(<2 \mathrm{~g} /$ dia $)$ como opção terapêutica aceitável para pacientes com dislipidemia combinada, mesmo sendo diabéticos (82).

\section{CONCLUSŌES}

Novos alvos de LDL-C e não HDL-C colesterol foram estabelecidos para pacientes com risco cardiovascular muito alto. Estas metas freqüentemente exigem grandes reduções nos níveis de LDL-C e não HDL-C, sendo necessárias estatinas mais potentes ou associação com drogas que diminuam a absorção de colesterol; ezetimiba é a droga de escolha desta classe terapêutica por apresentar menor freqüência de efeitos adversos.

Terapia hipolipemiante combinada também é importante no manuseio de hiperlipidemias combinadas que acometem freqüentemente indivíduos diabéticos e portadores de síndrome metabólica. Embora terapia redutora de LDL-colesterol com estatina promova redução significativa da mortalidade cardiovascular nestes pacientes, permanece ainda risco residual. Drogas como fibratos e niacina promovem adicional redução do risco cardiovascular. A associação de estatinas com fibratos pode aumentar o risco de hepatotoxicidade e miopatia; dentre os fibratos, o fenofibrato é claramente mais adequado em associação que o gemfibrozil, não havendo muitos dados disponíveis sobre a segurança da associação de estatinas com bezafibrato e ciprofibrato. Pacientes portadores de comprometimento renal ou hepático, idosos e aqueles necessitando múltiplas drogas não devem utilizar terapia combinada estatina-fibrato.
A associação estatina-niacina também é bastante eficaz na redução do risco cardiovascular em pacientes com hiperlipidemia combinada. Esta associação parece não aumentar muito o risco de miopatia, mas é limitada pelos efeitos colaterais da niacina, como flushing, e tendência à hiperglicemia e hiperuricemia. Flushing pode ser abrandado pelo uso noturno da niacina, precedido de ácido acetilsalicílico, sendo as preparações de liberação lenta mais bem toleradas (especialmente o Niaspan). Pacientes com doença péptica, hepatopatias, portadores de hiperuricemia ou gota não são bons candidatos ao uso de niacina. Diabéticos e pacientes que apresentem intolerância glicídica necessitam de especial atenção ao controle glicêmico e avaliação criteriosa da relação custo-benefício.

\section{REFERÊNCIAS}

1. Brunzell JD, Schrott HG, Motulsky AG, Bierman EL. Myocardial infarction in the familial forms of hypertriglyceridemia. Metabolism 1976;25:313-20.

2. Pajukanta P, Lilja HE, Sinsheimer JS, Cantor RM, Lusis AJ, Gentile $M$, et al. Familial combined hyperlipidemia is associated with upstream transcription factor 1 (USF1). Nat Genet 2004;36:371-6.

3. van der Vleuten $G M$, van Tits LJ, den Heijer M, Lemmers $H$, Stalenhoef AF, de Graaf J. Decreased adiponectin levels in familial combined hyperlipidemia patients contribute to the atherogenic lipid profile. J Lipid Res 2005;46:2398-404.

4. Xydakis $\mathrm{AM}$, Ballantyne $\mathrm{CM}$. Role of non-high-density lipoprotein cholesterol in prevention of cardiovascular disease: updated evidence from clinical trials. Curr Opin Cardiol 2003; 18:503-9.

5. Veerkamp MJ, de Graaf J, Stalenhoef AFH. Role of insulin resistance in familial combined hyperlipidemia. Arterioscler Thromb Vasc Biol 2005;25:1026-31.

6. Bredie SJ, Tack CJ, Smits P, Stalenhoef AF. Non-obese patients with familial combined hyperlipidemia are insulin resistant compared with their non-affected relatives. Arterioscler Thromb Vasc Biol 1997;17:1465-71

7. Karhapaa P, Voutilainen E, Kovanen PT, Laakso M. Insulin resistance in familial and non-familial hypercholesterolemia. Arterioscler Thromb 1993; 13:41-7

8. Mahley RW, Huang Y, Rall SC Jr. Pathogenesis of type III hyperlipoproteinemia (dysbetalipoproteinemia). Questions, quandaries, and paradoxes. J Lipid Res 1999; 40:1933-49.

9. National Cholesterol Education Program. Second report of the expert panel on detection, evaluation, and treatment of high blood cholesterol in adults (Adult Treatment Panel II). Circulation 1994;89:1333-445.

10. Executive Summary of the Third Report of the National Cholesterol Education Program (NCEP). Expert panel on detection, evaluation, and treatment of high blood cholesterol in adults (Adult Treatment Panel III). JAMA 2001:285:2486-97. 
11. Grundy SM, Cleeman JI, Merz CN, Brewer HB Jr, Clark LT, Hunninghake DB, et al; National Heart, Lung, and Blood Institute; American College of Cardiology Foundation; American Heart Association. Implications of recent clinical trials for the National Cholesterol Education Program Adult Treatment Panel III guidelines. Circulation 2004:110:227-39.

12. De Backer $G$, Ambrosioni E, Borch-Johnsen K, Brotons C, Cifkova R, Dallongeville J, et al; European Society of Cardiology; American Heart Association; American College of Cardiology. European guidelines on cardiovascular disease prevention in clinical practice. Third Joint Task Force of European and other Societies on Cardiovascular Disease Prevention in Clinical Practice. Atherosclerosis 2004; 173:381-91.

13. Heart Protection Study Collaborators Group: MRC/BHF heart protection study of cholesterol lowering with simvastatin in 20,536 high-risk individuals: a randomized placebo-controlled trial. Lancet 2002;360:7-22.

14. Cannon CP, Braunwald E, McCabe CH, Rader DJ, Rouleau JL, Belder $\mathrm{R}$, et al. Pravastatin or atorvastatin evaluation and infection therapy-thrombolysis in myocardial infarction 22 investigators. Intensive versus moderate lipid lowering with statins after acute coronary syndromes. N Engl J Med 2004;350: 1495-504.

15. Gordon DJ, Probstfield JL, Garrison RJ, Neaton JD, Castelli WP, Knoke JD, et al. High-density lipoprotein cholesterol and cardiovascular disease. Four prospective American studies. Circulation 1989;79:8-15.

16. Gordon T, Castelli WP, Hjortland MC, Kannel WB, Dawber TR. High density lipoprotein as a protective factor against coronary heart disease: The Framingham Study. Am J Med 1977;62:707-14.

17. Sharrett AR, Ballantyne CM, Coady SA, Heiss G, Sorlie PD, Catellier $\mathrm{D}$, et al. Coronary heart disease prediction from lipoprotein cholesterol levels, triglycerides, lipoprotein(a), apolipoproteins A-I and B, and HDL density subfractions: the Atherosclerosis Risk in Communities (ARIC) Study. Circulation 2001; 104:1 108-13.

18. Thompson MM, Reed SC, Cockerill GW. Therapeutic approaches to raising plasma HDL-cholesterol levels. Nat Clin Pract Cardiovasc Med 2004; 1:84-9.

19. Derby CA, Feldman HA, Bausserman LL, Parker DR, Gans $\mathrm{KM}$, Carleton RA. HDL cholesterol: trends in two southeastern New England communities, 1981-1993. Ann Epidemiol 1998;8:84-91.

20. Rohrer L, Hersberger M, von Eckardstein A. High density lipoproteins in the intersection of diabetes mellitus, inflammation and cardiovascular disease. Curr Opin Lipidol 2004; 15:269-78.

21. Davidson MH, Toth PP. Comparative effects of lipidlowering therapies. Prog Cardiovasc Dis 2004;47:73-104.

22. Liao, James K. Clinical implications for statin pleiotropy. Curr Opin Lipidol 2005; 16:624-9.

23. Buchwald $H$, Boen JR, Nguyen PA, Williams SE, Matts JP. Plasma lipids and cardiovascular risk: $\mathrm{A} P \mathrm{POS} \mathrm{CH}$ report. Program on the surgical control of the hyperlipidemias. Atherosclerosis 2001;154:221-7.

24. Jones PH, Davidson MH, Stein EA, Bays HE, McKenney JM, Miller E, et al; STELLAR Study Group. Comparison of the efficacy and safety of rosuvastatin versus atorvastatin, simvastatin, and pravastatin across doses (STELLAR* Trial). Am J Cardiol 2003;92:152-60.
25. Winkler K, Abletshauser C, Hoffmann MM, Friedrich I, Baumstark MW, Wieland $\mathrm{H}$, et al. Effect of fluvastatin slow-release on low density lipoprotein (LDL) subfractions in patients with type 2 diabetes mellitus: baseline LDL profile. J Clin Endocrinol Metab 2002:87:5485-90

26. Shepherd J, Cobbe SM, Ford I, Isles CG, Lorimer AR, Macfarlane PW, et al: West of Scotland Coronary Prevention Study Group. Prevention of coronary heart disease with pravastatin in men with hypercholesterolemia. N Engl J Med 1995;333:1301-7.

27. Ballantyne CM, Olsson AG, Cook TJ, Mercuri MF, Pedersen TR, Kjekshus J; Scandinavian Simvastatin Survival Study (4S) Group. Influence of low high-density lipoprotein cholesterol and elevated triglyceride on coronary heart disease events and response to simvastatin therapy in 4S. Circulation 2001; 104:3046-51.

28. Spach DH, Bauwens JE, Clark CD, Burke WG. Rhabdomyolysis associated with lovastatin and erythromycin use. West J Med 1991;154:213-5.

29. Nemeroff CB, DeVane L, Pollack BG. New antidepressants and the cytochrome P450 system. Am J Psychiatry 1996;153:311-20.

30. Miehalase EL. Update: clinically significant cytochrome P-450 drug interactions. Pharmacotherapy 1998;18:84112.

31. Venkatesan S, Cullen P, Pacy P, Halliday D, Scott J. Stable isotopes show a direct relation between VLDL apoB overproduction and serum triglyceride levels and indicate a metabolically and biochemically coherent basis for familial combined hyperlipidemia. Arterioscler Thromb 1993;13:1110-8.

32. Varady KA, Jones PJ. Combination diet and exercise interventions for the treatment of dyslipidemia: an effective preliminary strategy to lower cholesterol levels? J Nutr 2005; 135:1829-35.

33. Devi N. Antiretroviral therapy-induced hyperlipidemia. Int J STD AIDS 2005; 16(suppl 1):2-10.

34. Farmer JA, Torre-Amione $G$. Comparative tolerability of the HMG-CoA reductase inhibitors. Drug Saf 2000:23:197-213

35. Thompson PD, Clarkson P, Karas RH. Statin-associated myopathy. JAMA 2003;289:1681-90.

36. Ballantyne CM, Corsini A, Davidson $\mathrm{MH}$, Holdaas $\mathrm{H}$, Jacobson TA, Leitersdorf $E$, et al. Risk for myopathy with statin therapy in high-risk patients. Arch Intern Med 2003; 163:553-64.

37. Jamal SM, Eisenberg MJ, Christopoulos S. Rhabdomyolysis associated with hydroxymethylglutarylcoenzyme A reductase inhibitors. Am Heart J 2004; 147:956-65

38. Omar MA, Wilson JP. FDA adverse event reports on statin-associated rhabdomyolysis. Ann Pharmacother 2002;36:288-95.

39. Kobashigawa JA, Katznelson S, Laks $H$, Johnson JA, Yeatman $\mathrm{L}$, Wang $\mathrm{XM}$, et al. Effect of pravastatin on outcomes after cardiac transplantation. N Engl J Med1995;333:621-7.

40. Wenke K, Meiser B, Thiery J, Nagel D, von Scheidt W, Krobot $\mathrm{K}$, et al. Simvastatin initiated early after heart transplantation: 8-year prospective experience. Circulation 2003:107:93-7. 
41. Holdaas H, Fellstrom B, Jardine AG, Holme I, Nyberg G, Fauchald $\mathrm{P}$, et al. Assessment of LEscol in Renal Transplantation (ALERT) Study Investigators. Effect of fluvastatin on cardiac outcomes in renal transplant recipients: a multicentre, randomised, placebocontrolled trial. Lancet 2003;361:2024-31.

42. Kreisberg RA, Oberman A. Medical management of hyperlipidemia/dyslipidemia. J Clin Endocrinol Metab 2003:88:2445-61.

43. Kosoglou T, Statkevich $P$, Johnson-Levonas AO, Paolin $\mathrm{JF}$, Bergman AJ, Alton KB. Ezetimibe: A review of its metabolism, pharmacokinetics and drug Interactions. Clin Pharmacokinet 2005:44:467-94.

44. Gagne C, Bays HE, Weiss SR, Mata P, Quinto K, Melino M, et al. Efficacy and safety of ezetimibe added to ongoing statin therapy for treatment of patients with primary hypercholesterolemia. Am J Cardiol 2002; 90:1084-91.

45. Davidson MH, McGarry T, Bettis R, Melani L, Lipka LJ, LeBeaut $A P$, et al. Ezetimibe coadministered with simvastatin in patients with primary hypercholesterolemia. J Am Coll Cardiol 2002;40:2125-34.

46. Ballantyne C, Houri J, Notarbartolo A, Melani L, Lipka LJ, Suresh $\mathrm{R}$, et al. Effect of ezetimibe coadministered with atorvastatin in 628 patients with primary hypercholesterolemia: A prospective, randomized, double-blind trial. Circulation 2003:107:2409-15.

47. Melani L, Mills R, Hassman D, Lipetz R, Lipka L, LeBeaut A, et al. Efficacy and safety of ezetimibe coadministered with pravastatina in patients with primary hypercholesterolemia: A prospective, randomized, double-blind trial. Eur Heart J 2003:8:685-9.

48. Sullivan S. Ezetimibe complements fenofibrate in mixed hyperlipidaemia. Inpharma Weekly 2004;1467:13-4.

49. Westphal S, Wiens L, Guttler K, Dierkes J, Luley C. Chylomicron remnants of various sizes are lowered more effectively by fenofibrate than by atorvastatin in patients with combined hyperlipidemia. Atherosclerosis 2003: 171:369-77

50. Staels B, Auwerx J. Regulation of apo A-I gene expression by fibrates. Atherosclerosis 1998; 137:S19-23.

51. Vu-Dac N, Schoonjans K, Laine B, et al. Fibrates increase human apolipoprotein A-II expression through activation of the peroxisome proliferatoractivated receptor. Clin Invest 1995;96:741-50.

52. Bolibar I, von Eckardstein A, Assman G, et al. Shortterm prognostic value of lipid measurements for coronary events in patients with angina pectoris. Thromb Haemost 2000;84:955-60.

53. Fruchart JC. Peroxisome proliferator-activated receptor a activation and high-density lipoprotein metabolism. Am J Cardiol 2001;88(suppl):24N-9.

54. Lemieux I, Salomon H, Despres JP. Contribution of apoClll reduction to the greater effect of 12-week micronized fenofibrate than atorvastatin therapy on triglyceride levels and LDL size in dyslipidemic patients. Ann Med 2003:35:442-8.

55. Libby P. Inflammation in atherosclerosis. Nature 2002:420:868-74
56. Delerive P, Gervois P, Fruchart JC, Staels B. Induction of $\mathrm{kBa}$ expression as a mechanism contributing to the antiinflammatory activities of peroxisome proliferatoractivated receptor-alpha activators. J Biol Chem 2000:24:275:36703-7.

57. Wang TD, Chen WJ, Lin JW, Cheng CC, Chen MF, Lee YT. Efficacy of fenofibrate and simvastatin on endothelial function and inflammatory markers in patients with combined hyperlipidemia: Relations with baseline lipid profiles. Atherosclerosis 2003; 170:315-23.

58. Manninen $V$, Tenkanen $L$, Koskinen $P$, Huttunen JK, Manttari $M$, Heinonen OP, et al. Joint effects of serum triglyceride and LDL cholesterol and HDL cholesterol concentrations on coronary heart disease risk in the Helsinki Heart Study. Implications for treatment. Circulation 1992;85:37-45.

59. Robins SJ, Collins D, Wittes JT, Papademetriou V, Deedwania PC, Schaefer EJ, et al. VA-HIT Study Group. Veterans Affairs High-Density Lipoprotein Intervention Trial. Relation of gemfibrozil treatment and lipid levels with major coronary events. JAMA 2001;285:1586-9.

60. The BIP Study Group. Secondary prevention by raising HDL cholesterol and reducing triglycerides in patients with coronary artery disease. The Bezafibrate Infarction Prevention Study. Circulation 2000; 102:21-7.

61. Ericsson CG, Hamsten A, Nilsson J, Grip L, Svane B, de Faire U. Angiographic assessment of effects of bezafibrate on progression of coronary artery disease in young male postinfarction patients. Lancet 1996; $347: 849-53$

62. Frick $M H$, Syvänne $M$, Nieminen MS, Kauma $H$, Majahalme S, Virtanen V, et al. Prevention of the angiographic progression of coronary and vein-graft atherosclerosis by gemfibrozil after coronary bypass surgery in men with low levels of $\mathrm{HDL}$ cholesterol. Circulation 1997:96:2137-43.

63. Diabetes Atherosclerosis Intervention Study Investigators. Effect of fenofibrate on progression of coronary artery disease in type 2 diabetes: The Diabetes Atherosclerosis Intervention Study: A randomized study. Lancet 2001;357:905-10.

64. Grundy SM, Vega GL, Yuan Z, Battisti WP, Brady WE Palmisano J. Effectiveness and tolerability of simvastatin plus fenofibrate for combined hyperlipidemia (the SAFARI trial). Am J Cardiol 2005:95:462-8.

65. Gaist D, Rodriguez LA, Huerta C, Hallas J, Sindrup SH. Lipid-lowering drugs and risk of myopathy: a populationbased follow-up study. Epidemiology 2001;12:565-9.

66. Broeders N, Knoop C, Antoine M, Tielemans C, Abramowicz D. Fibrate-induced increase in blood urea and creatinine: is gemfibrozil the only innocuous agent? Nephrol Dial Transplant 2000; 15:1993-9.

67. Hottelart C, El Esper N, Rose F, Achard JM, Fournier A. Fenofibrate increases creatininemia by increasing metabolic production of creatinine. Nephron 2002:92:536-41.

68. Shitara $Y$, Sato H, Sugiyama Y. Evaluation of drug-drug interaction in the hepatobiliary and renal transport of drugs. Annu Rev Pharmacol Toxicol 2005;45:689-723.

69. Jones $\mathrm{PH}$, Davidson $\mathrm{MH}$. Reporting rate of rhabdomyolysis with fenofibrate + statin versus gemfibrozil + any statin. Am J Cardiol 2005;95:120-2. 
70. Graham DA, Staffa JA, Shatin D, Andrade SE, Schech $\mathrm{SD}$, Grenade LL, et al. Incidence of hospitalized rhabdomyolysis in patients treated with lipid-lowering drugs. JAMA 2004;292:2585-90.

71. Backman JT, Kyrklund C, Neuvonen M, Neuvonen PJ. Gemfibrozil greatly increases plasma concentrations of cerivastatin. Clin Pharmacol Ther 2002;72:685-91.

72. Shek A, Ferrill MJ. Statin-fibrate combination therapy. Ann Pharmacother 2001;35:908-17.

73. Pauciullo $\mathrm{P}$, Borgnino $\mathrm{C}$, Paoletti R, Mariani M, Mancini M. Efficacy and safety of a combination of fluvastatin and bezafibrate in patients with mixed hyperlipidaemia (FACT study). Atherosclerosis 2000; 150:429-36.

74. Gavish D, Leibovitz E, Shapira I, Rubinstein A. Bezafibrate and simvastatin combination therapy for diabetic dyslipidaemia: efficacy and safety. J Intern Med 2000;247:563-9.

75. Fruchart JC, Brewer HB Jr, Leitersdorf E. Consensus for the use of fibrates in the treatment of dyslipoproteinemia and coronary heart disease. Fibrate Consensus Group. Am J Cardiol 1998;81:912-7.

76. Xydakis AM, Ballantyne CM. Combination therapy for combined dyslipidemia. Am J Cardiol 2002;90:21 K-29K.

78. Goldberg A, Alagona P, Capuzzi DM, Guyton J, Morgan $\mathrm{JM}$, Rodgers J, et al. Multiple-dose efficacy and safety of an extended-release form of niacin in the management of hyperlipidemia. Am J Cardiol 2000;85: 1 100-5.

79. Capuzzi DM, Guyton JR, Morgan JM, Goldberg AC, Kreisberg RA, Brusco OA, et al. Efficacy and safety of an extended-release niacin (Niaspan): A long-term study. Am J Cardiol 1998;82:74U-81U.

80. Schlierf $G$, Dorow E. Diurnal patterns of triglyceride, free fatty acids, blood sugar, and insulin during carbohydrate-induction in man and their modification by nocturnal suppression of lipolysis. J Clin Invest $1973: 52: 732-40$

81. Canner PL, Furberg CD, Terrin ML, et al. Niacin decreases myocardial infarction and total mortality in patients with metabolic syndrome: Results from the Coronary Drug Project. J Am Coll Cardiol 2003:39:291A.
82. Grundy SM, Vega GL, McGovern ME, Tulloch BR, Kendall DM, Fitz-Patrick D, et al. Efficacy, safety, and tolerability of once-daily niacin for the treatment of dyslipidemia associated with type 2 diabetes. Arch Intern Med 2002: 162:1568-76.

83. Shepherd J, Betteridge J, Van Gaal L. Nicotinic acid in the management of dyslipidaemia associated with diabetes and metabolic syndrome: a position paper developed by a European Consensus Panel. Curr Med Res Opin 2005;21:665-82.

84. Carlson LA, Rosenhamer $\mathrm{G}$. Reduction of mortality in the Stockholm Ischaemic Heart Disease Secondary Prevention Study by combined treatment with clofibrate and nicotinic acid. Acta Med Scand 1988;223:405-18.

85. Zema MJ. Gemfibrozil, nicotinic acid and combination therapy in patients with isolated hypoalphalipoproteinemia: A randomized, open-label crossover study. J Am Coll Cardiol 2000;35:640-6.

86. Brown G, Albers JJ, Fisher LD, Schaefer SM, Lin JT, Kaplan $\mathrm{C}$, et al. Regression of coronary artery disease as a result of intensive lipid lowering therapy in men with high levels of apolipoproteína B. N Engl J Med 1990;323:1289-98.

87. Brown BG, Zhao $X Q$, Chait A, Fisher LD, Cheung MC, Morse JS, et al. Simvastatin and niacin, antioxidant vitamins, or the combination for the prevention of coronary disease. N Engl J Med 2001;345:1583-92.

88. Elam MB, Hunninghake DB, Davis KB, Garg R, Johnson C, Egan $D$, et al. Effect of niacin on lipid and lipoprotein levels and glycemic control in patients with diabetes and peripheral arterial disease: the ADMIT study: A randomized trial. Arterial Disease Multiple Intervention Trial. JAMA 2000;284:1263-70.

\section{Endereço para correspondência:}

Ísio Schulz

Disciplina de Endocrinologia

Hospital das Clínicas / Faculdade de Medicina

Universidade de São Paulo (HC-FMUSP)

Av. Dr. Enéas de Carvalho Aguiar 155, $8^{\circ}$ andar - Bloco 3a

05403-000 São Paulo, SP

E-mail: ischulz@uol.com.br 